376 PILOT EVALUATION OF RISK ASSESSMENT AND ENHANCED PROTOCOLS REGARDING CONTACTS AT AN INTERNATIONAL PROFESSIONAL GOLF EVENT

\begin{abstract}
1,2Patrick Robinson, ${ }^{28,8,9,10}$ Andrew Murray, ${ }^{3,4}$ Volker Scheer, ${ }^{5}$ Graeme Close, ${ }^{6,7}$ Denis Kinane. 'Edinburgh Orthopaedics, Royal Infirmary of Edinburgh, Edinburgh, UK; ${ }^{2}$ European Tour Performance Institute, Virginia Water, UK; ${ }^{3}$ Health Science Department, Universidad a Distancia de Madrid, Madrid, Spain; ${ }^{4}$ Ultra Sports Science Foundation, Pierre Benite, France; ${ }^{5}$ Research Institute for Sport and Exercise Sciences, Liverpool John Moores University, Liverpool, UK; ${ }^{6}$ School of Dental Medicine, University of Berne, Berne, Switzerland; 'Medical and Scientific Department, Cignpost Diagnostics, Farnborough, UK; ${ }^{8}$ Sport and Exercise, University of Edinburgh, Edinburgh, UK; ${ }^{9}$ Medical and Scientific Department. The RandA, St. Andrews, UK; ${ }^{10}$ Medical and Scientific Department. The International Golf Federation, Lausanne, UK
\end{abstract}

10.1136/bjsports-2021-IOC.343

Background Following conversations with the International Chief Medical Officer Group for Sport, the World Health Organisation Mass Gatherings team, and host public health teams, a risk assessment and managed risk approach was piloted for professional golf at the Gran Canaria Lopesan Open $17^{\text {th }}$ to $25^{\text {th }}$ April 2021.

Objective The aim of this study was to assess if a risk-assessed approach to contact tracing was practical to undertake at a major sporting event while also enabling a safe environment for those participating.

Design Prospective cohort study

Setting Professional golf event.

Patients (or Participants) Attendees of event.

Interventions (or Assessment of Risk Factors) All participants required a minimum of one negative RT-PCR test prior to travelling to each tournament. High risk contacts were isolated for 10 days. Moderate risk contacts received education regarding enhanced medical surveillance, had daily rapid antigen testing for 5 days, with RT-PCR day 5, mandated mask use, and access to outside space for work purposes only. Low risk contacts received rapid antigen testing every 48 hours and PCR testing on day 5.

Main Outcome Measurements RT-PCR positive test.

Results A total of 550 persons were accredited and were required to undergo RT-PCR testing before the event. Two of these tests were positive $(0.36 \%)$. Of these, Case 1 had one high, 23 moderate, and 48 low risk contacts. Case 2 did not have any significant travel history within 2 days of positive test, and had one high risk contact. There were no further positive tests on site in the wider cohort of attendees, from a total of 872 RT-PCR and 198 rapid antigen tests.

Conclusions This pilot study showed it is practical, feasible, and well accepted to provide enhanced (daily) virus testing and risk-mitigating measures at a professional golf event.

\section{TEST-RETEST, INTRA- AND INTER-RATER RELIABILITY OF THE REACTIVE BALANCE TEST: A NEUROCOGNITIVE FUNCTIONAL TEST TO EVALUATE ADAPTABILITY WITHIN INJURY RISK PROFILING}

${ }^{1}$ Bruno Tassignon, '1Jo Verschueren, 'Jonas De Wachter, 'Alexandre Maricot, 1,3Kevin De Pauw, ${ }^{2}$ Evert Verhagen, ${ }^{1,3}$ Romain Meeusen. ${ }^{1}$ Human Physiology and Sports Physiotherapy Research Group, Faculty of Physical Education and Physiotherapy, Vrije Universiteit Brussel, Brussels, Belgium; ${ }^{2}$ Amsterdam Collaboration on Health and Safety in Sports, Department of Public and Occupational Health, Amsterdam UMC, Vrije Universiteit Amsterdam, Amsterdam Movement Sciences, Amsterdam, Netherlands; '35trategic Research Program Exercise and the Brain in Health and Disease: the added value of Human-Centered Robotics, Vrije Universiteit Brussel, Brussels, Belgium

10.1136/bjsports-2021-IOC.344
Background Balance tests are often selected to screen for injury risk, but only evaluate pre-planned movements. Recently, adaptability was put forward as a key driver in injury prevention. Adaptability is defined as athletes' ability to adequately adapt their responses under a comprehensive variety of conditions. The reactive balance test (RBT) is designed as a neurocognitive functional test that integrates environmental perception and decision-making to evaluate adaptability while maintaining unilateral balance.

Objective To determine test-retest, intra- and inter-rater reliability of the RBT in healthy recreational athletes.

Design Test-retest reliability study design.

Setting Primary prevention in clinical setting.

Participants Twenty-one healthy recreational athletes (age $=22$ \pm 1 years, height $=175 \pm 9 \mathrm{~cm}$, weight $=69 \pm 7 \mathrm{~kg}$ ).

Interventions Two experimental trials were separated by an average of $33 \pm 15$ days. During experimental trials participants performed the Y-balance test (to determine maximal reach distances) four times and the RBT once. The LED lights of the RBT were set at $80 \%$ of the maximal reach distance on each axis of the Y-balance test. The RBT consists out of 36 randomised stimuli.

Main Outcome Measurements Outcome measures of the RBT are accuracy and visuomotor response time. Intraclass correlation coefficients (ICC), standard errors of measurement and prediction, and minimal detectable difference were calculated.

Results Excellent intra- rater reliability was observed for visuomotor response time (ICC: 0.992, [0.981;0.997]) and accuracy (ICC: 0.925, [0.827;0.969]). Excellent inter-rater reliability was also observed for both visuomotor response time (ICC: 0.978, [0.946;0.991]) and accuracy (ICC: 0.920, [0.803;0.968]). Test-retest reliability for visuomotor response time could be considered good (ICC: 0.831, [0.629;0.928]), while moderate test-retest reliability was found for accuracy (ICC: 0.706 , [0.420;0.820]).

Conclusions Our results indicate that overall test-retest, intraand inter-rater reliability of the RBT was moderate to excellent. Thus, the RBT possesses acceptable reliability to use in group level analyses. Future research should further determine the clinimetric properties of the RBT in specific populations and research the RBT along the sport injury continuum.

\section{MAXIMISING THE RELEVANCE AND DISSEMINATION OF THE IOC MEDICAL CONSENSUS STATEMENTS: KEY STAKEHOLDER'S PERCEPTIONS OF THE IOC CONSENSUS STATEMENTS IN A DEVELOPING COUNTRY (SOUTH AFRICA)}

${ }^{4}$ Marelise Badenhorst, 'Lauren Fortington, ${ }^{2}$ Caroline Bolling, ${ }^{2}$ Evert Verhagen, ${ }^{3}$ Carolyn Emery, ${ }^{5}$ Martin Schwellnus, ${ }^{3}$ Kati Pasanen, ${ }^{4}$ Wayne Derman, ${ }^{1}$ Caroline Finch. ${ }^{1}$ Australian Centre for Research into Injury in Sport and its Prevention (ACRISP), School of Medical and Health Sciences, Edith Cowan University, Joondalup, Australia; ${ }^{2}$ Amsterdam Collaboration on Health and Safety in Sports, Department of Public and Occupational Health, Amsterdam UMC, Amsterdam, Netherlands; ${ }^{3}$ Sport Injury Prevention Research Centre, University of Calgary, Calgary, Canada; ${ }^{4}$ Insitute of Sport and Exercise Medicine, University of Stellenbosch, Cape Town, South Africa; ${ }^{5}$ Sport, Exercise Medicine and Lifestyle Institute (SEMLI), University of Pretoria, Pretoria, South Africa

\subsection{6/bjsports-2021-IOC.345}

Background The IOC Sports Medical and Scientific Commission has supported the development and dissemination of sports medicine consensus statements for athlete health. 
Evidence on the relevance and dissemination of these statements is important for the development of future statements. Objective The objective of this project was to investigate the relevance and dissemination of the IOC consensus statements among sports medicine professionals directly involved in Olympic athlete health in a developing setting (South Africa). Design Qualitative case study.

Methods Semi-structured interviews, document analysis and field-notes were utilised. Seven sports medicine professionals interviewed.

Results Awareness around consensus statement topics and perceived access to the statements was limited in South Africa, especially for clinicians who are not currently active within an academic or research setting. In terms of relevance, participants described the importance of practical relevance of the statements, emphasizing the need for inclusion of the athlete's voice and diversity in skills, experience and context of the consensus statement authors. Participants also described the need to align format and content of the information according to the target audience. The usability and utilisation of the statements were determined by the perception of relevance at the time, within the specific context, as well as the ability to provide a practical message. Healthcare inequities, poorly resourced national federations, as well as general resource and time restrictions when managing athletes (reactive versus proactive management) were also considered as barriers to utilisation.

Conclusions The statements were perceived by South African sports medicine professionals as being relevant and beneficial for the management of athlete health. However, issues around awareness, access, usability, and practical application in a developing country were also raised.

\section{MAXIMISING THE RELEVANCE AND DISSEMINATION OF THE IOC MEDICAL CONSENSUS STATEMENTS: KEY STAKEHOLDER'S PERCEPTIONS OF THE IOC MEDICAL CONSENSUS STATEMENTS IN A DEVELOPED COUNTRY (AUSTRALIA)}

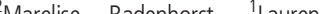
${ }^{2}$ Wayne Derman, ${ }^{4}$ Evert Verhagen, ${ }^{3}$ Martin Schwellnus, ${ }^{5}$ Carolyn Emery, ${ }^{1}$ Caroline Finch. ${ }^{1}$ Australian Centre for Research into Injury in Sport and its Prevention (ACRISP), School of Medical and Health Sciences, Edith Cowan University, Joondalup, Australia: ${ }^{2}$ Insitute of Sport and Exercise Medicine, University of Stellenbosch, Cape Town, South Africa; ${ }^{3}$ Sport, Exercise Medicine and Lifestyle Institute (SEMLI), University of Pretoria, Pretoria, South Africa; ${ }^{4}$ Amsterdam Collaboration on Health and Safety in Sports, Department of Public and Occupational Health, Amsterdam UMC, Amsterdam, Netherlands; ${ }^{5}$ Sport Injury Prevention Research Centre, University of Calgary, Calgary, Canada

10.1136/bjsports-2021-IOC.346

Background The IOC Medical and Scientific commission's mission is to provide a guiding reference for sports organisations on issues relating to the protection of the health of athletes. One strategy to achieve this mission is through supporting the development of consensus statements.

Objective This study investigates the relevance and dissemination of the IOC consensus statements among sports medicine professionals directly involved in Olympic athlete health in a developed setting (Australia).

Design Qualitative case study.

Methods Semi-structured interviews, document analysis and field notes were utilised. Seven sports medicine professionals were interviewed.
Results For clinicians (not active in an academic/research setting), knowledge of the statements was limited; invitation to the study was the first awareness of the statements. Participants in leadership roles (with an academic/research component) were generally more familiar with some, but not all, statements. Participants identified a wealth of competing information sources through professional networks in health and sport. Subsequently, participants tend to consider these other avenues as first access point for information. Where statements were identified and used, there were several benefits reported, particularly drawn from the perceived leadership, trust, brand and reach of the IOC. While there is no formal adoption process for the information contained within a consensus statement, where identified as relevant, the information is shared amongst peer networks, via email or face-to-face meetings/seminars. There was agreement that the IOC has a role in the development and dissemination of the statements. However, interviewees also stated that information that is current and tailored to the target audiences (clinicians, coaches or athletes) could enhance the IOC's objective of guiding reference on athlete health.

Conclusions A wealth of information is available to sports medicine professionals in Australia. Therefore, to enhance relevance and dissemination in a developed country, the IOC consensus statements must remain current and be tailored to target audiences.

\section{THE ENGLISH INSTITUTE OF SPORT'S MENTAL HEALTH JOURNEY}

Samuel Cumming, Craig Ranson. English Institute of Sport, Manchester, UK

\subsection{6/bjsports-2021-IOC.347}

Background Following a number of reviews into Olympic and Paralympic sport in the UK it was identified that mental health would be an area of focus for the Tokyo cycle. This led to development and implementation of a specific mental health strategy consisting of four pillars; education, provision, communication and assurance.

Implementation of a strategy to better-support the mental health of all people within the UK's High Performance System.

Design Retrospective service evaluation of the implementation of UK Sport's Mental Health Strategy, using statistics relating to two of the strategy's four pillars - Education and Provision.

Setting Athletes and staff who are part of the UK's High Performance Sports System.

Participants Athletes and staff from the UK's High Performance Sports System.

Intervention A Mental Health Expert Panel was established in late 2018 to provide world-leading expert advice and guidance to the system on matters relating to mental health. In addition, an elite sport-specific mental health education programme was designed and delivered between 2019 and 2020

Main outcome measurements The primary outcome measures focus on usage of and feedback on the newly implemented initiatives (i.e. the Mental Health Expert Panel and education programme).

Results We observed significant uptake of mental health services (e.g. requests to access EIS Mental Health Expert Panel; 